\title{
Feasibility of Immuno-TRITC Labeling in Integrated 3D CLEM
}

Sergey V. Loginov ${ }^{1}$, Alexandra V. Agronskaia ${ }^{1}$, Wally H. Müller ${ }^{2}$, Elly G. van Donselaar ${ }^{3}$, Job Fermie $^{1,3}$, Judith Klumperman ${ }^{3}$, Gerhard A. Blab ${ }^{1}$ and Hans C. Gerritsen ${ }^{1}$

1. Soft Condensed Matter and Biophysics, Debye Institute and EMME $\Phi$, Utrecht University, Utrecht, The Netherlands.

2. Cryo-EM, Chemistry department, Science Faculty, Utrecht University, Utrecht, The Netherlands.

3. Department of Cell Biology, University Medical Center Utrecht, Utrecht, The Netherlands.

Three dimensional Correlative Light and Electron Microscopy (3D CLEM) by an integrated Confocal Laser Scanning Microscope (CLSM) and a Focused Ion Beam / Scanning Electron Microscope (FIB/SEM) is a powerful tool for biological studies. The integration of these two modalities calls for the specific fluorescent labeling which must survive the heavy metal staining required for the FIB/SEM imaging. In this work the feasibility of immuno-TRITC labeling of cell samples suitable for the $\mathrm{FIB} / \mathrm{SEM}$ is shown.

The FIB/SEM facilitates 3D imaging of biological samples with resolution up to several nanometers in all three dimensions. However, the acquisition of a volume of $10 \times 10 \times 10 \mu \mathrm{m}^{3}$ with such fine resolution takes up to several days. The comparatively fast and low resolution CLSM can be used to guide the much slower but higher resolution FIB/SEM to regions of interest inside thick samples. The integration of the two modalities in one setup provides the best possible registration accuracy.

Integration of the CLSM in the FIB/SEM requires samples simultaneously fluorescently labeled for CLSM and stained for FIB/SEM imaging. The challenge here is to retain dye fluorescence in a very heavy-metal rich resin embedded samples prepared for the FIB/SEM imaging.

Though existing protocols describe preservation of the fluorescence signal in resin embedded heavy metal stained samples, they are either limited to non-immunolabeled fluorescence staining [1] or do not work in the vacuum conditions [2,3]. Nevertheless, TRITC fluorescence under vacuum conditions was reported previously for integrated 2D CLEM [4]. The transition from 2D to 3D CLEM introduces an additional challenge: the heavy metals staining now surrounds the dye from all directions.

We investigated the possibility of the use of the TRITC dye for immunolabeling in integrated 3D CLEM. As the test system, cultivated SKBR3 cells were used. The cells were chemically fixed and thereafter labeled first with Herceptin ${ }^{\circledR}$ and subsequently with secondary antibodies conjugated with TRITC. At this stage of the preparation of the SKBR3 cells, wide-field fluorescence imaging was performed. Next, the cells were treated according to a modified FIB/SEM sample preparation protocol [5] which consisted of heavy metal staining - osmium tetroxide, uranyl acetate and lead aspartate - and subsequent thin layer plastification into Spurr's resin. Wide-field fluorescence imaging was performed again to measure the remaining TRITC dye signal. To control the specificity of the labeling, SKBR3 cells were prepared otherwise identically, but without the use of Herceptin ${ }^{\circledR}$.

The resulting images of the cells were analyzed to quantify the fluorescence signal of the label. In the Fig. 1, typical images of the cells before (Fig. 1a) and after (Fig. 1b) the heavy metal staining and embedding are shown. Average intensity of the cells is shown on Fig. 1c. The sample prepared for the 
3D CLEM loses fluorescent signal up to one order of magnitude, however the specific signal is still two times higher than background.

3D CLEM measurements were performed by the use of a home-made CLSM system and a FEI Scios FIB/SEM. The results (Fig.2) show that the fluorescence CLSM image can serve to guide the FIB/SEM. Clear correlation between fluorescence signal and membrane folds is observed.

References:

[1] MS Lucas et al, Methods in Molecular Biology 1117 (2014), p. 593.

[2] W Kukulski et al, Methods in Cell Biology 111 (2012), p. 235.

[3] CJ Peddie et al, Ultramicroscopy 143 (2014), p. 3.

[4] MA Karreman et al, Journal of Structural Biology 180 (2012), p. 382.

[5] AG Bittermann et al, European Microscopy Conference (2012).
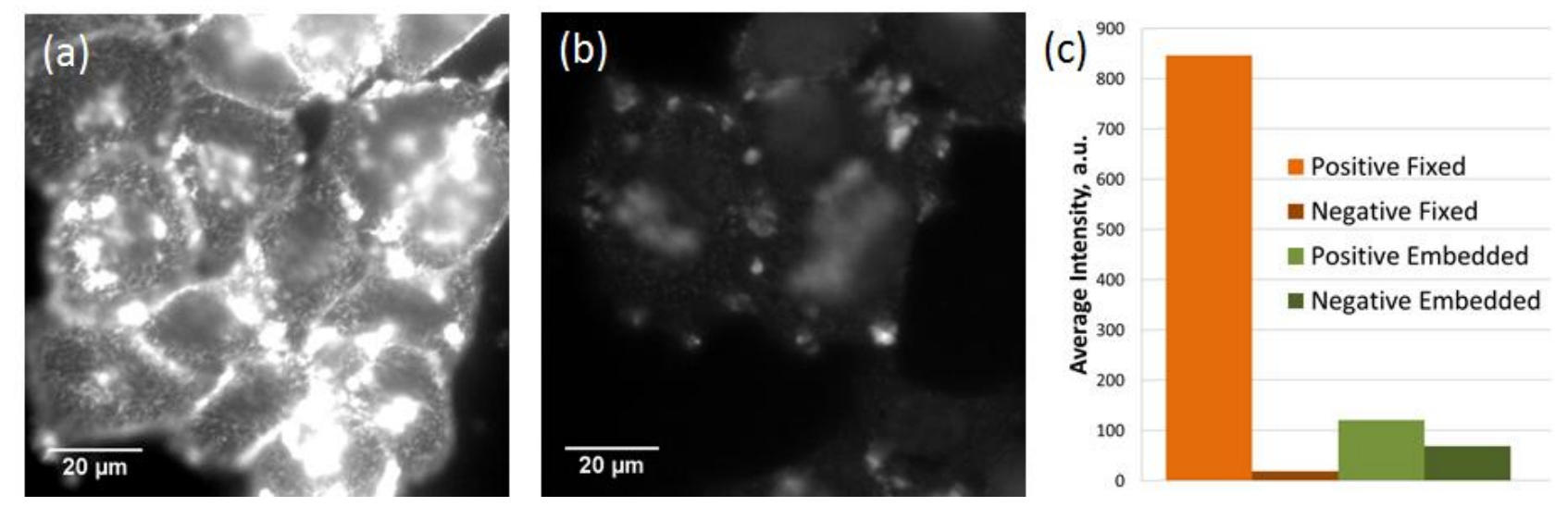

Figure 1. Typical wide-field images of immunolabeled SKBR3 cells (a) before heavy-metal staining and (b) after preparation for the FIB/SEM imaging; both (a) and (b) use the same intensity scaling; (c) Average intensities (population of $\sim 100$ cells) of the stained and thin layer resin embedded cells compared to control (no primary antibody) stained and thin layer resin embedded samples.
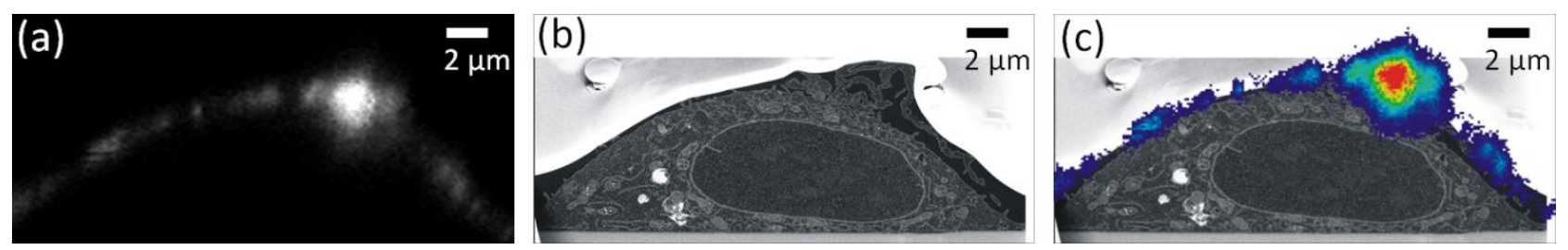

Figure 2. Images of a thin layer resin embedded SKBR3 cell (a) Virtual (xz) cross-section of the CLSM 3D stack. (b) One of the corresponding FIB/SEM slices. The cell morphology is clearly visible. (c) The overlay of (a) and (b) images, the higher intensity of the fluorescence corresponds to the membrane fold. 